\title{
Research Article \\ Impact of Background Traffic on Speech Quality in VoWLAN
}

\author{
Peter Počta, Peter Kortiš, and Martin Vaculík \\ Department of Telecommunications, FEE, University of Žilina, Univerzitná 1, 01026 Žilina, Slovakia
}

Received 24 April 2007; Accepted 26 July 2007

Recommended by Stavros Kotsopoulos

This paper describes measurements of the impact of background traffic on speech quality in an environment of WLANs (IEEE 802.11). The simulated background traffic consists of three types of current traffics in telecommunication networks such as data transfer service, multimedia streaming service, and Web service. The background traffic was generated by means of the accomplished Distributed Internet Traffic Generator (D-ITG). The impact of these types of traffic and traffic load on speech quality using the test sequence and speech sequences is the aim of this paper. The assessment of speech quality is carried out by means of the accomplished Perceptual Evaluation of Speech Quality (PESQ) algorithm. The proposal of a new method for improved detection of the critical conditions in wireless telecommunication networks from the speech quality point of view is presented in this paper. Conclusion implies the next application of the method of improved detection of critical conditions for the purpose of algorithms for link adaptation from the speech quality point of view in an environment of WLANs. The primary goal of these algorithms is improving speech quality in the VoWLAN connections, which are established in the competent link.

Copyright (C) 2007 Peter Počta et al. This is an open access article distributed under the Creative Commons Attribution License, which permits unrestricted use, distribution, and reproduction in any medium, provided the original work is properly cited.

\section{INTRODUCTION}

Voice over Internet Protocol (VoIP), the transmission of packetized voice over internet protocol networks, has gained much attention in recent years. It is expected to carry more and more voice traffic for its cost-effective service. However, the current Internet, which was originally designed for data communications, provides best-effort service only, posing several technical challenges for real-time VoIP applications. Speech quality is impaired by packet loss, delay, and jitter. Assessment of perceived speech quality in the IP networks becomes an imperative task to manufacturers as well as service providers.

Speech quality is judged by human listeners and hence it is inherently subjective. The Mean Opinion Score (MOS) test, defined by ITU-T P.800 [1], is widely accepted as a norm for speech quality assessment. However, such subjective test is expensive and time-consuming. It is impractical for frequent testing such as routine network monitoring.

Objective test methods have been developed in recent years. They can be classified into two categories: signalbased methods and parameter-based methods. Signal-based methods use two signals as the input to the measurements, namely, the reference signal and the degraded signal, which is the output of the system under test. They identify the audible distortions based on the perceptual domain representation of two signals incorporating human auditory models. These methods include Perceptual Speech Quality Measure (PSQM), Measuring Normalizing Blocks (MNB), Perceptual Analysis Measurement System (PAMS), and Perceptual Evaluation of Speech Quality (PESQ). Among them, PSQM and PESQ [2] were standardized by ITU-T as P.861 and P.862, respectively. Parameterbased methods predict the speech quality through a computation model instead of using real measurements. The typical model is the E-model as defined by ITU-T recommendation G.107. The E-model includes a set of parameters characterizing the end-to-end voice transmission as its input, and the output can be transformed into a MOS scale for prediction.

The algorithm PSQM is based on comparison of the power spectrum of the corresponding sections of the reference and the degraded signals. The results of this algorithm more correlate with the results of listening tests, in comparison with E-model. At present, this algorithm is no longer used because of a raw time alignment. Instead of it the algorithm PESQ is rather used. The algorithm PESQ is facilitated with very fine time alignment and one single interruption being also taken into account in the calculation of MOS. It is possible to use PESQ in mobile networks as well as in networks based on packet transmission. The disadvantages include impossibility to use it for codec with data rate lower 
than $4 \mathrm{kbps}$, and higher calculation load caused by recursions employed in the algorithm.

To provide person-to-person (instead of place-to-place) connections anywhere and anytime, the Internet is expected to penetrate the wireless domain. One very promising wireless network is the Wireless Local Area Network (WLAN), which has shown the potential to provide high-rate data services at low cost over local area coverage. Working in the license-exempted $2.4 \mathrm{GHz}$ industrial, scientific, and medical (ISM) frequency band, the IEEE 802.11b WLAN and IEEE $802.11 \mathrm{~g}$ WLAN offer a data rate up to $11 \mathrm{Mbps}$ and $54 \mathrm{Mbps}$, respectively, while IEEE 802.11a WLAN and European Telecommunications Standard Institute (ETSI) Hiperlan/2 can support data rates up to $54 \mathrm{Mbps}$ in the $5 \mathrm{GHz}$ frequency band. Nowadays, the work on upcoming IEEE 802.11 WLAN standard is in progress. Its publication is currently expected in September, 2008 [3]. The IEEE 802.11n will be able to support a data rate up to $248 \mathrm{Mbps}$ ( 2 streams) in the $5 \mathrm{GHz}$ or $2.4 \mathrm{GHz}$ frequency band. As a wireless extension to the wired Ethernet, WLANs typically cover a small geographic area, in hotspot local areas where the traffic intensity is usually much higher than in other areas.

The promising VoIP technology and wide deployment of WLANs are assumed to drive the application of Voice over WLAN (VoWLAN), which is expected to experience a dramatic increase in the near future [4].

One major challenge for VoWLAN is Quality of Service (QoS) provisioning. Originally designed for high-rate data traffic, WLANs may experience bandwidth inefficiency when supporting delay-sensitive and low-rate voice traffic. Hence, it is essential to enhance the QoS support capability of current WLAN standards, such as the most popular IEEE 802.11 standard. The assessment of speech quality in the current WLAN standards is very desirable opportunity for evaluation, verification, and testing of enhanced QoS methods for supporting voice services in this type of very fast developing wireless telecommunication networks.

Some works have studied the effects of packet loss and jitter on speech quality. Particularly, [5-7] examined these effects in the MOS domain for certain packet loss rate and packet sizes in the environment of IP networks. Only in [8] was examined the effect of packet loss, jitter, and number of users on perceived speech quality based on PESQ algorithm in the wireless networks, especially in 3G networks. In [9], the effect of free bandwidth on VoIP performance in IEEE $802.11 \mathrm{~b}$ WLAN was investigated. The E-model has been used for the purpose of speech quality prediction in IEEE 802.11b WLAN. The close relationship between wireless bandwidth utilization and call quality was found. A lot of works have been performed in the area of evaluation of IEEE 802.11e Enhanced Distribution Coordination Access (EDCA) for enhanced VoWLAN performance, for example, $[10,11]$. In [10], the legacy IEEE 802.11 Distribution Coordination Function (DCF) and the IEEE 802.11 EDCA were compared to show that the EDCA can provide differentiated channel access among different priority traffics. In [11], the impact of adjustment Arbitration InterFrame Space (AIFS), Contention Window (CW), and Transmission Opportunity (TXOP) on the delay and the throughput of two competing saturated stations were examined. The scheme for prioritizing voice was suggested for IEEE 802.11 infrastructure mode networks. This scheme is based on increasing AIFS value used by other stations. Some works focus on the improving of current QoS support methods, for example, [12]. In [12], a new Medium Access Control (MAC) protocol is proposed for QoS support in WLAN. The protocol is an alternative to the recent enhancement IEEE 802.11e. A new priority policy provides the system with better performance by simulating Time Division Multiple Access (TDMA) functionality.

Here, we focus on the impact of background traffic on speech quality of transmission sequences in the environment of IEEE 802.11 networks. The background traffic was generated by means of Distributed Internet Traffic Generator (DITG) [13]. The simulated background traffic consists of three types of current traffics. The current traffics are: data transfer service, multimedia streaming service, and Web service. Increasing traffic load causes the increasing jitter and packet loss. In general, speech quality drops with increasing packet loss and jitter. The impact of these types of traffic and traffic load on speech quality is studied in this paper. The speech quality is assessed by means of the accomplished PESQ algorithm. The proposal of a new method for improved detection of the critical conditions in telecommunication networks from the speech quality point of view is presented at the end of this paper.

The rest of the paper is organized as follows. Section 2 briefly reviews the limitations of IEEE 802.11 in supporting of voice and IEEE 802.11e EDCA. Section 3 describes the measurement scenario. Section 4 presents the measurement results. In Section 5, we propose a method for improved speech quality-centered detection of critical conditions in telecommunication networks. Section 6 concludes the paper and suggests some future studies.

\section{VOICE SERVICE IN IEEE 802.11}

\subsection{Limitations of IEEE 802.11 in supporting voice service}

As a real-time application, VoWLAN is delay-sensitive but can tolerate a certain level of packet loss. Hence, delay and jitter are the main QoS measures. Each voice packet should be transmitted within delay bounds. Also, the jitter (i.e., variation of voice packet delay) should be carefully controlled as it may degrade speech quality more severely than delay. Traditionally, an appropriately designed jitter buffer is an effective way to deal with jitter and make the voice understandable [14]. Therefore, the investigation of the impact of network performance parameters change (jitter, packet loss) on speech quality is the main goal of this paper. The change of network performance parameters is realized by means of background traffic. The investigation of these influences allows designing the methods for improving speech quality in wireless networks.

As the most popular WLAN standard, IEEE 802.11 defines a mandatory DCF and an optional centralized Point Coordination Function (PCF). DCF is based on Carrier Sense Multiple Access with Collision Avoidance (CSMA/CA), 
where collision is resolved by binary exponential backoff. The optional Request-To-Send (RTS)/Clear-To-Send (CTS) dialog can also be applied to further deal with the hidden terminal problem. Mainly designed for data transmission, DCF does not take into account the delay-sensitive nature of realtime services. On the other hand, with PCF, Contention-Free Period (CFP) and a Contention Period (CP) alternate periodically. During CFP, when polled, a station gets permission to transmit its data frames. The main drawbacks of PCF include bandwidth waste when two stations in the same Basic Service Set (BSS) (which is composed of an Access Point (AP) and number of stations associated with the AP) try to communicate with each other, uncontrolled transmission time of a polled station, and unpredictable CFP start time [15].

In order to support applications with QoS requirements, the IEEE 802.11e standard [16] enhances the original IEEE 802.11 MAC sublayer by introducing the Hybrid Coordination Function (HCF), which includes two medium access methods: Enhanced Distributed Channel Access (EDCA) and HCF Coordination Channel Access (HCCA). In this paper, we will focus only on the EDCA method because a major part of currently available APs is enabled only for the EDCA method.

\subsection{IEEE 802.11e EDCA}

EDCA is designed to enhance the DCF mechanism and to provide a distributed access method that can support service differentiation among traffic categories. The service differentiation is provided by assigning different contention parameters to different Access Category (AC). A QoS station can support at most eight user priorities, which are mapped into four ACs. Each AC contends channel access with different AIFS and CW settings. Compared with DCF, where DCF InterFrame Space (DIFS) is used as the common InterFrame Space (IFS) for a station to access the channel, EDCA uses different AIFS for each AC to achieve the access differentiation, where the AIFS for a given AC is defined as

$$
\operatorname{AIFS}[\mathrm{AC}]=\mathrm{SIFS}+\mathrm{AIFSN} \times \sigma .
$$

The AIFSN denotes the number to differentiate the AIFS for each AC, and $\sigma$ is the time interval of a slot for IEEE 802.11 standard, which is determined according to the physical medium used. The AC with the smallest AIFS has the highest priority. EDCA assigns smaller CWs to ACs with higher priorities to bias the successful transmission probability in favor of high-priority ACs in a statistical sense. Indeed, the initial CW size $\left(\mathrm{CW}_{\min }\right)$ can be set differently for different priority ACs, yielding higher priority ACs with smaller $\mathrm{CW}_{\text {min. }}$. Figures 1 and 2 illustrate these EDCA parameters and the access procedure, respectively.

In the EDCA, both the physical carrier sensing and the virtual sensing methods are similar to those in the DCF. However, there is a major difference in the countdown procedure when the medium is determined to be idle. In the EDCA, after the AIFS period, the backoff counter decreases by one at the beginning of the last slot of the AIFS (shown as the crossed time slot in Figure 2), while in the DCF, this is

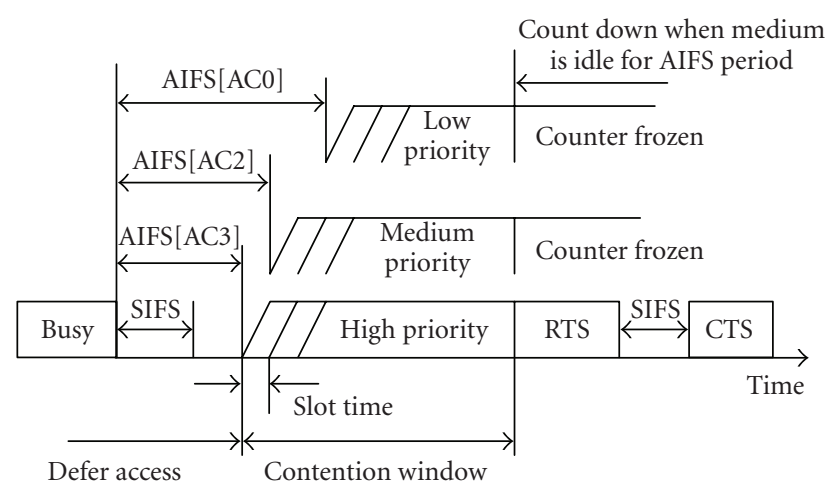

FIgURE 1: IEEE 802.11e EDCA mechanism parameters.

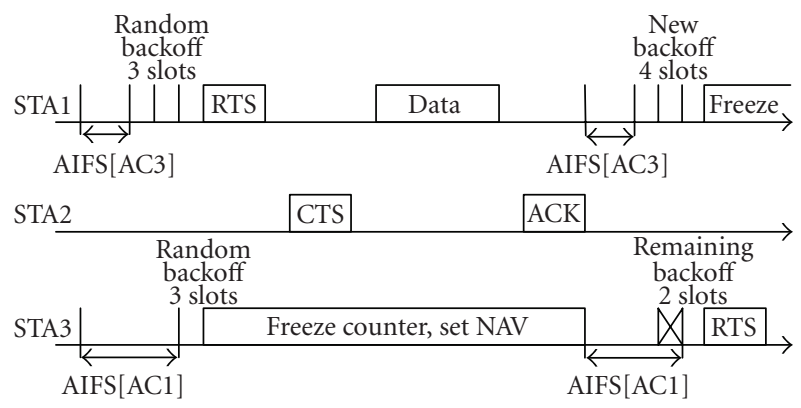

FIgURE 2: IEEE 802.11e EDCA channel access procedure.

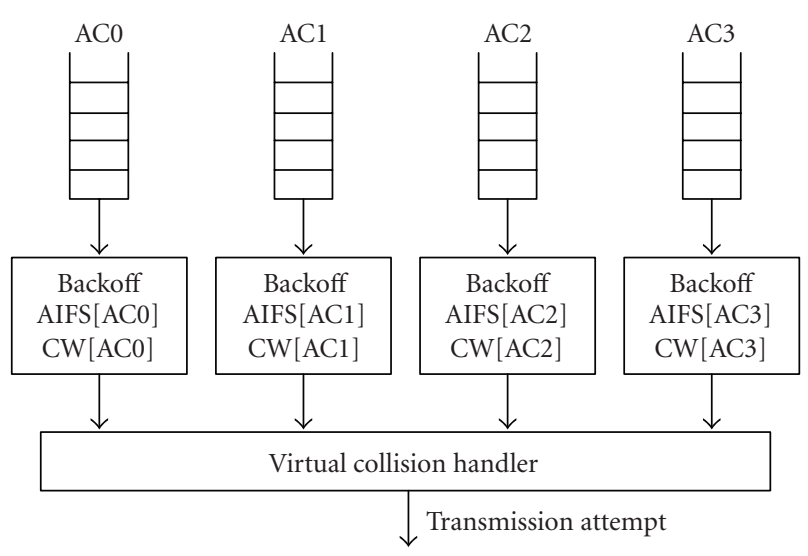

Figure 3: ACs and virtual collision.

done at the beginning of the first time slot interval following the DIFS period $[10,16,17]$.

For a given station, traffics of different ACs are buffered in different queues as shown in Figure 3. Each AC within a station behaves like a virtual station; it contends for access to the medium and independently starts its backoff after sensing that the medium is idle for at least AIFS period. When a collision occurs among different ACs within the same station, the higher-priority AC is granted the opportunity for physical transmission, while the lower-priority AC suffers from a virtual collision, which is similar to a real collision outside the station $[10,16,17]$. 


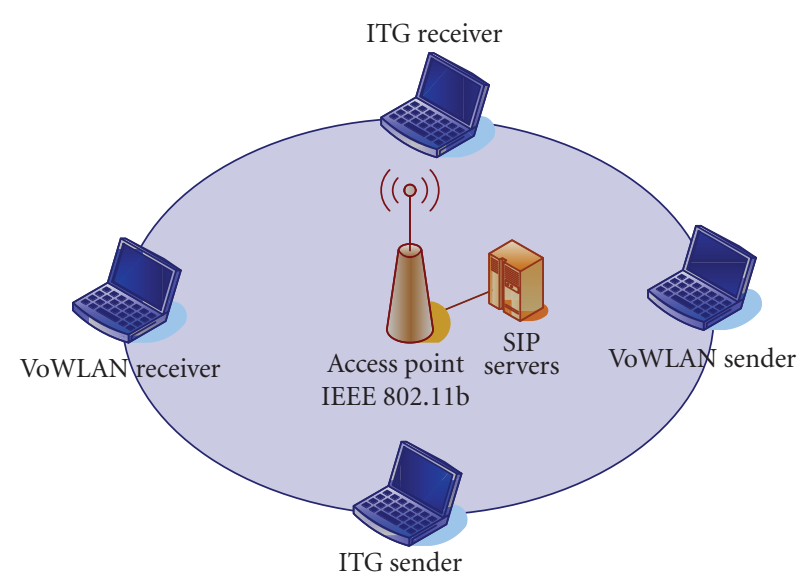

FIgURE 4: Measurement setup.

IEEE 802.11e also defines a TXOP limit as the interval of time during which a particular station has the right to initiate transmissions. During an EDCA TXOP, a station may be allowed to transmit multiple data frames from the same AC with a Short InterFrame Space (SIFS) gap between an ACK and the subsequent data frame $[10,16,17]$.

\section{MEASUREMENTS}

\subsection{Experimental setup}

One-way VoWLAN session was established between two wireless hosts (VoWLAN sender and VoWLAN receiver), via the AP, in IEEE 802.11b WLAN (see Figure 4).

Two wireless stations (ITG sender and ITG receiver) equipped with the accomplished D-ITG were used to generate and receive background traffic. ITG sender generated the User Datagram Protocol (UDP) and Transmission Control Protocol (TCP) packets of the length 1024 bytes. Background traffic is described in Section 3.3. Voice traffic was generated using VoIP clients. Session Initiation Protocol (SIP) was used for established VoWLAN connections. For the measurement we chose the ITU-T G.729A encoding scheme [18]. In the measurement, each frame was encapsulated into a packet in turn, corresponding to a packet size of 10 milliseconds. Adaptive jitter buffer, packet loss concealment, and voice activity detector are implemented using VoIP clients.

The ITU-T recommendation P.862.3 [19] recommends to use a sequence in the range from 8 -second to 30 -second duration for the purpose of speech quality measurement. We decided to use the 30-second sequence for the needs of our measurements. The 30-second sequence enables to realize the precise speech quality measurement. The duration of each measurement was set equal to the length of sequence. The measurements were performed for six different testing conditions. The sequences described in Section 3.2 were utilized for transmission through the given VoWLAN connection. Finally, PESQMOS was measured by PESQ algorithm.

The adjusting of station positions and antennas was being performed until signal strength and link quality achieved by all stations were roughly similar. All stations had fixed
TABLE 1: EDCA parameters used for measurements.

\begin{tabular}{ccccc}
\hline $\mathrm{AC}$ & $\mathrm{CW}_{\min }$ & $\mathrm{CW}_{\max }$ & AIFSN & $\mathrm{TXOP}(\mathrm{b})[\mu \mathrm{s}]$ \\
\hline Voice & 3 & 7 & 1 & 3264 \\
Video & 7 & 15 & 1 & 6016 \\
Best Effort & 15 & 1023 & 3 & 0 \\
Background & 15 & 1023 & 7 & 0 \\
\hline
\end{tabular}

position (no mobility) during all measurements. The signal strength and link quality were kept in the range from $85 \%$ to $100 \%$ (excellent) for all performed measurements.

As we aim to use IEEE 802.11 technology for the transmission of real-time services, EDCA has to be applied to support this type of services. This fact is reviewed in Section 2. The EDCA parameters are described in Table 1. The values of CW and AIFSN represent the number of time slots; each slot is of 20 -microsecond duration. The beacon frame was periodically transmitted every 1000 milliseconds.

\subsection{Description of sequences}

Two types of sequence were used for the purpose of the measurement. The first type of sequence is the test sequence composed of simple signals. Speech sequences are the second type of sequence. The speech sequences are composed from speech records.

\subsubsection{Description of test sequence}

The test sequence consists of non-speech-like (fully artificial) signals. These signals are defined in ITU-T recommendation P.501 [20] which divides them into deterministic and random signals. Development of the method for more precise detection of the critical conditions in the telecommunication networks from the speech quality point of view was our motivation for using simple signals. The duration of the test sequence is set to 30 seconds. The test sequence is composed of the following signals introduced and evaluated in [21]:

(i) sinusoidal signal of frequencies $300,800,1000,1700$, 2400, $3000 \mathrm{~Hz}$,

(ii) square bipolar signal of frequencies 300, 400, 500, 600, 635, $670 \mathrm{~Hz}$,

(iii) Gaussian white noise with $\mu=0$ and $\delta=0.0001 ; 0.001$; $0.005 ; 0.01 ; 0.025 ; 0.05$.

The principle of the creation of the final test sequence is based on arranging the parts of the test sequence, which are shown in Figures 5 and 6. The final test sequence consists of six sections. Each section consists of five parts. The arrangement shown in Figure 5 is used once and subsequently the arrangement shown in Figure 6 is used four times to form the first section of final test sequence. The arrangement shown in Figure 6 is used five times to form the other sections of the final test sequence. The signals step by step have got the values defined above; for example, in the second section of the test sequence (from 5 seconds to 10 seconds), the signals have the following values: square bipolar signal $f=400 \mathrm{~Hz}$, Gaussian 


\begin{tabular}{|c|c|c|}
\hline $\begin{array}{c}\text { Square } \\
\text { bipolar signal } \\
(f=300 \mathrm{~Hz})\end{array}$ & $\begin{array}{c}\text { Gaussian white } \\
\text { noise } \\
(\delta=0.0001)\end{array}$ & $\begin{array}{c}\text { Sinusoidal } \\
\text { signal } \\
(f=300 \mathrm{~Hz})\end{array}$ \\
\hline \multicolumn{3}{|c|}{$1 \mathrm{~s}$} \\
\hline
\end{tabular}

Figure 5: Initial part of test sequence.

\begin{tabular}{|c|c|c|}
\hline $\begin{array}{c}\text { Sinusoidal } \\
\text { signal } \\
(f=300 \mathrm{~Hz})\end{array}$ & $\begin{array}{c}\text { Gaussian white } \\
\text { noise } \\
(\delta=0.0001)\end{array}$ & $\begin{array}{c}\text { Square } \\
\text { bipolar signal } \\
(f=300 \mathrm{~Hz})\end{array}$ \\
\hline \multicolumn{3}{|c|}{$1 \mathrm{~s}$} \\
\hline
\end{tabular}

Figure 6: Second part of test sequence.

white noise $\delta=0.001$, and sinusoidal signal $f=800 \mathrm{~Hz}$. The values of the signals in the first section of the test sequence (from 0 second to 5 seconds) are the same as those in Figures 5 and 6 . The test sequence was stored in 16-bit, $8000 \mathrm{~Hz}$ linear PCM.

The arrangement of test sequence was derived from the criteria given by ITU-T recommendations for speech quality measurements such as P.862.3. The very important requirement is that the sequence for speech quality measurements includes active speech and silence periods. In ITU-T recommendation P.862.3 is defined that the sequence should be active in the range from $40 \%$ to $80 \%$ of its duration. The active interval of proposed test sequence is about $66 \%$ of its duration. We decided to emulate active interval by means of simple (non-speech-like) signals such as sinusoidal signal and square bipolar signal. The silence period was modelled by means of Gaussian white noise.

The choice of the test sequence arrangement for intrusive measurement of Voice Transmission Quality of Service (VTQoS) was published in [22]. The optimization of the test sequence for ITU-T G.729 encoding scheme was published in [23].

It was found that the test sequence composed of simple signals is more sensitive to the transmission impairments (jitter, packet loss, etc.) in environment of IP network than a sequence composed of speech samples. Hence, such test sequence is more suitable for more precise detection of the qualitative changes in the IP networks. We compared these two types of sequences in [24].

\subsubsection{Description of speech sequences}

The speech sequences selection should follow the criteria given by ITU-T recommendation P.830 [25] and ITU-T recommendation P.800 [1]. The speech sequences should include bursts separated by silence periods. They are normally of 1-3 seconds long, although this does vary considerably between languages. Certain types of voice activity detectors are sensitive only to silent periods that are longer than 200 milliseconds. Also the speech sequences should be ac-

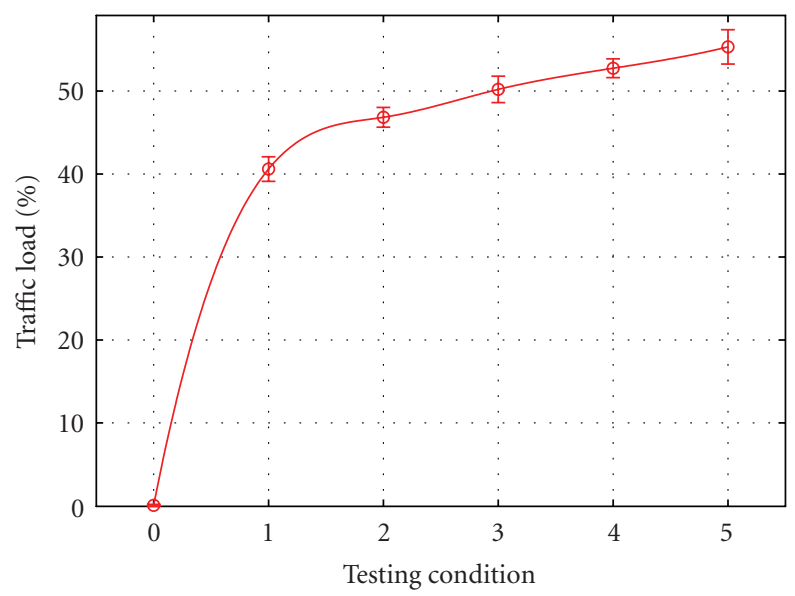

FIgure 7: Traffic load for given testing conditions. The vertical bars show $95 \%$ CI (derived from 10 measurements) for each testing condition. The testing condition numbers correspond to Table 2.

tive for $40 \%-80 \%$ of their duration. The speech sequences are composed of speech records. In our experiments, these speech records come from a Slovak speech database. In each set, two female and two male speech utterances were used. The speech sequences, which are of 30 -second long with $57 \%$ average value of active speech interval, were stored in 16-bit, $8000 \mathrm{~Hz}$ linear PCM.

\subsection{Background traffic}

Background traffic has been generated by D-ITG. The primary goal of background traffic is to simulate standard traffic that appears in WLANs, which includes data transfer via Hypertext Transfer Protocol (HTTP) and File Transfer Protocol (FTP), multimedia streams for real-time applications.

The simulated background traffic includes three types of communication.

(i) "Data transfer service", which includes FTP and other nonspecified services, is represented as information stream with constant bit rate based on TCP;

(ii) "Multimedia streaming service" represents real-time multimedia applications and therefore is based on information stream with constant bit rate. The UDP is used in this case;

(iii) "Web service" that is simulated as a sequence of separated data bursts with Poisson distribution of packet rate. The active period of burst is 400 milliseconds and the bursts appear periodically every two seconds. TCP was used for the purpose of this service.

The measurements have been performed for six different testing conditions. The selected bit rates of the three abovementioned types of communication and average traffic load of background traffic are described in Table 2 and Figure 7. The calculation of average traffic load was based on the $100 \%$ channel rate, which is $11 \mathrm{Mbps}$ for IEEE $802.11 \mathrm{~b}$ technology. 
TABLE 2: Performance evaluation of testing conditions.

\begin{tabular}{ccccc}
\hline Testing condition & $\begin{array}{c}\text { Data transfer service } \\
{[\mathrm{Mbps}]}\end{array}$ & $\begin{array}{c}\text { Multimedia streaming service } \\
{[\mathrm{Mbps}]}\end{array}$ & $\begin{array}{c}\text { Web service } \\
{[\mathrm{Mbps}]}\end{array}$ & $\begin{array}{c}\text { Average traffic load } \\
{[\%]}\end{array}$ \\
\hline 0 & 0 & 0 & 0 & 0 \\
1 & 2 & 2.5 & 0.5 & 40.7 \\
2 & 2.25 & 2.82 & 0.56 & 0.61 \\
3 \\
4 & 2.5 & 3.14 & 0.68 & 57.1 \\
5 & 2.75 & 3.45 & 0.74 & 53.5 \\
\hline
\end{tabular}

The simulation of the multimedia streaming service was carried out focusing on the impact of the service traffic on speech quality. Note that D-ITG does not allow the simulation of the multimedia streaming service using Real-time Transport Protocol (RTP), but the RTP-based streaming service has the same impact on speech quality as the streaming using UDP. The aim of this measurement was to investigate how these types of traffic and traffic load affect speech quality. The traffic load was measured by means of Wireshark network analyzer [26].

\subsection{Assessment of speech quality}

PESQMOS was evaluated by the PESQ metric [2], which is the recent ITU-T standard for objective speech quality assessment. PESQ combines merits of PAMS and PSQM99 (an updated version of PSQM), and adds new methods for transfer function equalization and averaging distortions over time. It can be used in wider range of network conditions, and gives higher correlation with subjective tests and the other objective algorithms $[2,27,28]$. Unlike the conversational model, PESQ is a listening-only model; the degraded sample is timealigned with the reference sample during preprocessing. The PESQMOS values do not reflect the effects of delay on speech quality.

\section{MEASUREMENT RESULTS}

The measurement was independently performed 10 times under the same testing conditions. The PESQMOS results were averaged out and the standard deviation was kept within 0.07 PESQMOS for the test sequence and 0.15 PESQMOS for the speech sequences.

Figure 10 shows the measurement results for the test sequence and speech sequences. The graphs represent the dependence of PESQMOS change on the testing conditions. The testing conditions represent a few types of network conditions. Each network condition is described by traffic load. The increasing traffic load causes jitter and also packet loss to increase. In general, speech quality drops with increasing packet loss and jitter. Figure 7 shows the traffic load for given testing conditions. The transmission rates for given testing conditions are described in Table 2. Figure 11 shows the measurement results for the speech sequences. We can see some differences in the impact of background traffic on speech quality among the individual speech sequences in Figure 11 . It is caused by a different arrangement of the speech

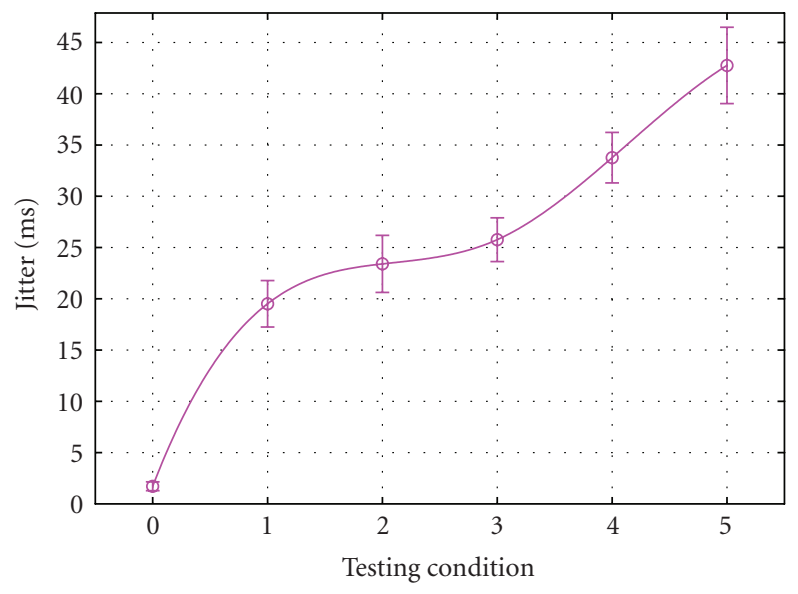

FIGURE 8: Impact of background traffic on average value of jitter in VoWLAN connections. Other detailed descriptions of Figure 7 apply appropriately.

sequences as well as by differences in duration of their active speech intervals among speakers. The impact of background traffic on the jitter (delay variation) and packet loss in VoWLAN connections is shown in Figures 8 and 9. The 1550 voice packets were approximately transmitted during one 30-second long VoWLAN connection. The average value of jitter ranged from 1.72 to 42.76 milliseconds and the total packet loss ranged from $0.81 \%$ to $23.03 \%$ for these measurements. The total packet loss consists of two components. The first component is the lost packets and the second component is the dropped packets.

Figure 10 represents only average values separately for female and male speech sequences. It can be seen that the test sequence has smoother characteristic than the speech sequences. From the speech quality point of view, the test sequence responds to network performance parameters change (jitter, packet loss, etc.) more sensitively than the speech sequences do. The effect of network performance parameters change causes higher difference in energy of the particular FFT points by using the stationary spectra signals such as sinusoidal signal and square bipolar signal. That is a reason of the increased sensitivity of the test sequence to the network performance parameters change. The enhanced sensitivity allows for carrying out the detection of qualitative changes in telecommunication networks more precisely. We aim to use it for the development of a method for improved speech 


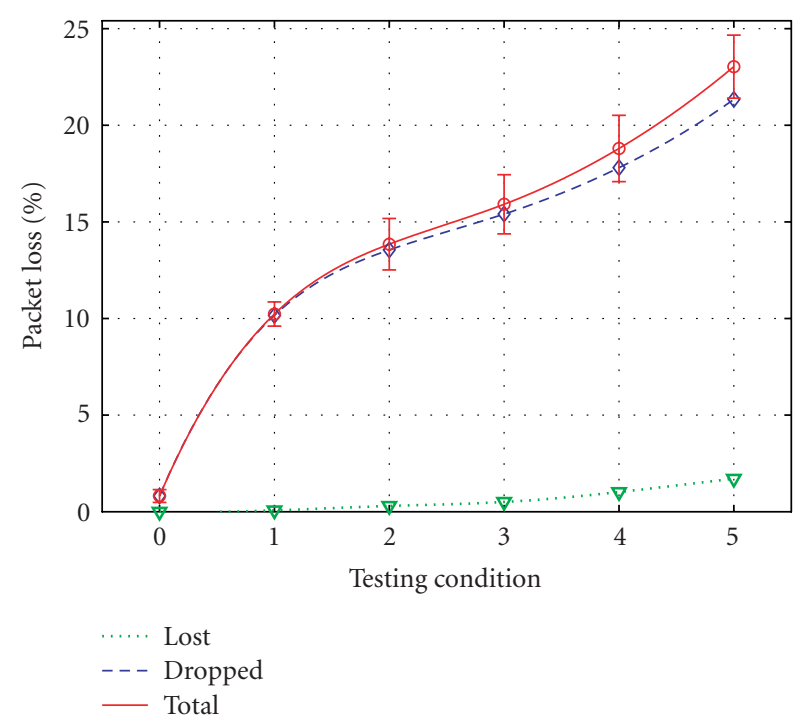

FIgURE 9: Impact of background traffic on packet loss in VoWLAN connections. Other detailed descriptions of Figure 7 apply appropriately.

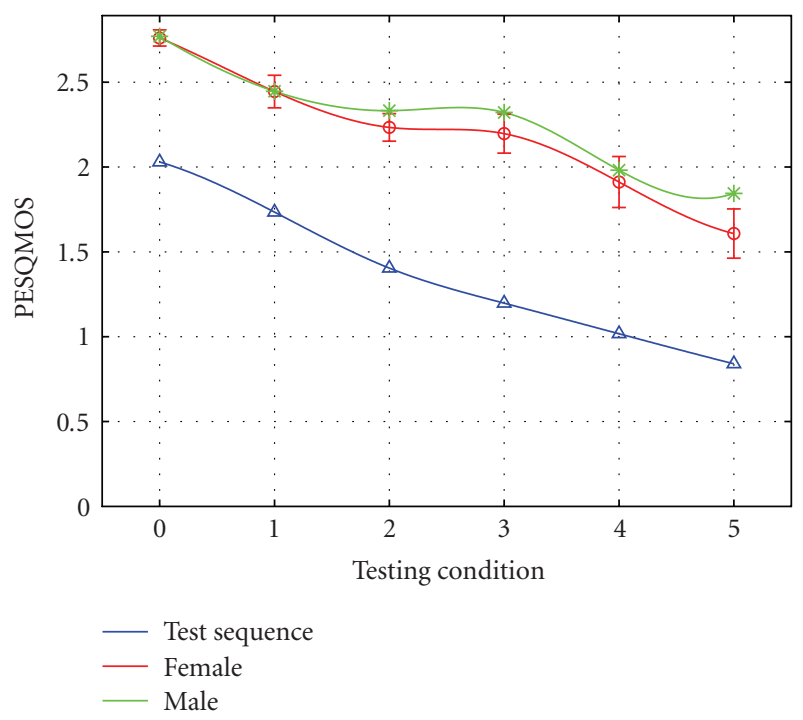

Figure 10: Impact of background traffic on speech quality of speech sequences and test sequence. Other detailed descriptions of Figure 7 apply appropriately.

quality-based detection of critical conditions in telecommunication networks.

\section{PROPOSAL OF METHOD FOR IMPROVED DETECTION OF CRITICAL CONDITIONS IN WIRELESS NETWORKS FROM THE SPEECH QUALITY POINT OF VIEW}

The proposal of the method for improved speech qualitycentered detection of critical conditions in telecommunication networks follows the experiment described in this paper. Such improved detection allows for realizing earlier response

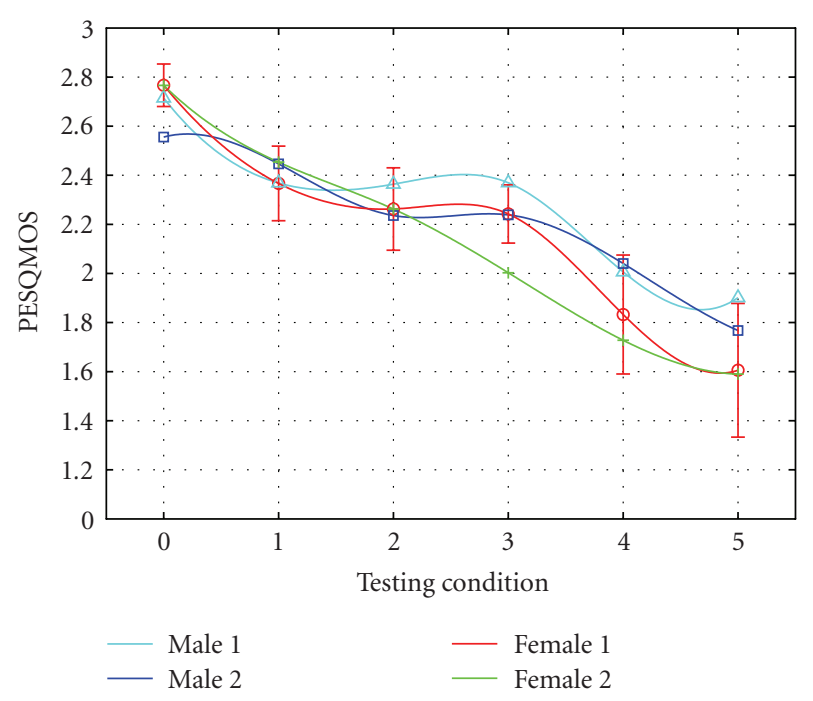

FIGURE 11: Impact of background traffic on speech quality of speech sequences. Other detailed descriptions of Figure 7 apply appropriately.

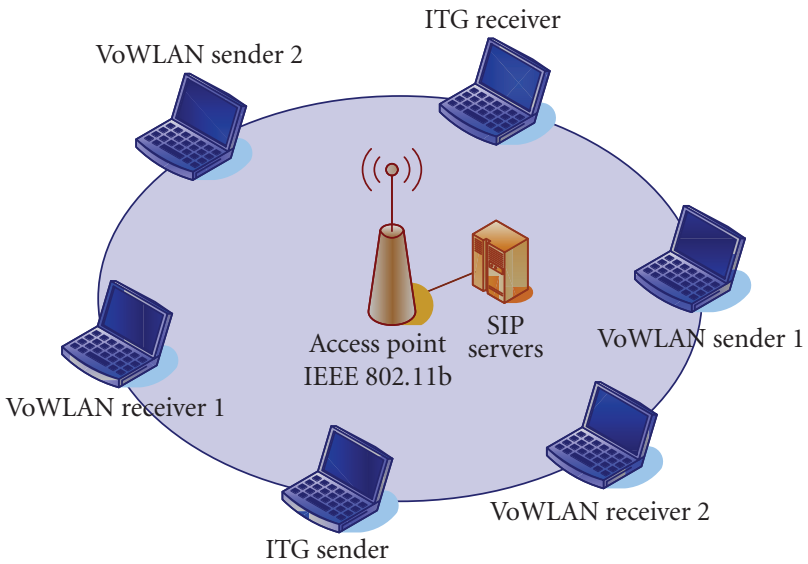

Figure 12: A typical network scenario in the environment of WLANs.

to the change of network performance parameters and it may avoid the impairment of speech quality. Figure 12 represents a typical network scenario in the environment of WLANs. VoWLAN connection no. 1 is used for the real VoWLAN transmission, and VoWLAN connection no. 2 is used for the purpose of improved detection of critical conditions in wireless telecommunication networks. This method is based on the intrusive measurement of VTQoS. The test sequence is transmitted through the VoWLAN connection no. 2. ITG stations represent the data traffic stations. The principle of the improved detection of the critical conditions is based on the simple scheme.

The measurement by using the test sequence is carried out in successive 30-second steps and the duration of the measurement is set to 30 seconds. The PESQMOS value is computed for each measurement. The PESQMOS is used as a threshold parameter for the network conditions. The 
TABLE 3: Decision level for the relevant network conditions.

\begin{tabular}{cc}
\hline PESQMOS value & The network condition \\
\hline$>1.275$ & Standard \\
$<1.275$ & Critical \\
\hline
\end{tabular}

threshold value for network conditions is set to 1.275 PESQMOS for the test sequence. This critical decision threshold has been derived from the results, which were obtained by the measurements described in this paper.

As seen from the measurement results (Figure 10), the PESQMOS value 1.275 for the test sequence corresponds to about $48 \%$ value of traffic load. The PESQMOS values of all the speech sequences (Figure 11) rapidly decline for the traffic load exceeding $48 \%$. The jitter and packet loss parameters (Figures 8 and 9) increase fast above $48 \%$ of the traffic load value. Table 3 figures out the decision level for the relevant network conditions.

When the PESQMOS value for test sequence is higher than the threshold value, the network is situated in the standard network conditions from the speech quality point of view. It means that speech quality is kept within a tolerable range and the adjustment of network parameters (e.g., AIFS, CW, packet size, etc.) for low-priority services is not necessary. The critical network conditions are expected when the PESQMOS value for test sequence is below the threshold value. The impairment of speech quality is expected and the adjustment of network parameters for low-priority services is required. The acceptable speech quality can not be expected when the adjustment of the network parameters is omitted. The network parameters will be adjusted by means of upcoming algorithms for link adaptation from the speech quality point of view as a future extension of this method. These algorithms are outlined in the conclusion.

\section{CONCLUSION AND FUTURE WORK}

This paper investigated the impact of background traffic on speech quality in VoWLAN applications. Different traffic testing conditions were used for the purpose of the measurements. Each testing condition consists of the three types of current traffics, which exist in the telecommunication networks. The current traffics are data transfer service, multimedia streaming service, and Web service. The results show that test sequence responds to the change of the network performance parameters (jitter, packet loss, etc.) more sensitively than the speech sequences from the speech quality point of view. It allows for performing an improved detection of qualitative changes in telecommunication networks. We propose the method for such speech quality-centered detection of critical conditions in wireless networks. In the future, we aim to expand this method to algorithms for link adaptation from the speech quality point of view in the environment of IEEE 802.11 networks. The primary goal of these algorithms is improving speech quality in VoWLAN connections, which are established in the competent link. The first type of the algorithm will be based on the fragmentation of data packets. The fragmentation of the large datagrams shall create packets of a size small enough to allow for satisfying the delay requirements of the delay-sensitive traffic. Small delay-sensitive packets could be interleaved between fragments of the large datagram. The second type of the algorithm will be based on prioritizing voice by means of increasing a station's AIFS value. The scheme for prioritizing voice, which was suggested in [11], is based on increasing AIFS value used by other stations. Increasing a station's AIFS value results in an increased delay after every transmission on network before that station can continue decrementing its counters. This scheme can be used to protect a voice call against large numbers of data stations, maintaining throughput, mean delays, and delay distributions in a range where high voice call quality can be expected. We suppose that the increasing AIFS value will have an impact on speech quality. Future work will also focus on developing and verifying these algorithms for such speech quality-centered link adaptation.

\section{REFERENCES}

[1] ITU-T Rec. P.800, "Methods for subjective determination of transmission quality," International Telecommunications Union, Geneva, Switzerland, 1996.

[2] ITU-T Rec. P.862, "Perceptual evaluation of speech quality," International Telecommunications Union, Geneva, Switzerland, 2001.

[3] Official IEEE 802.11 working group project timelines, http://grouper.ieee.org/groups/802/11/Reports/802.11_ Timelines.htm.

[4] "Voice over wireless LAN: 802.11x hears the call for wireless VoIP," Market research rep., in-stat, April 2002.

[5] L. A. R. Yamamoto and J. G. Beerends, "Impact of network performance parameters on the end-to-end perceived speech quality," in Proceedings of Expert ATM Traffic Symposium, Mykonos, Greece, September 1997.

[6] B. Duysburgh, S. Vanhastel, B. De Vreese, C. Petrisor, and P. Demeester, "On the influence of best-effort network conditions on the perceivedspeech quality of VoIP connections," in Proceedings of the 10th International Conference on Computer Communications and Networks (ICCCN '01), pp. 334339, Scottsdale, Ariz, USA, October 2001.

[7] L. Ding and R. A. Goubran, "Assessment of effects of packet loss on speech quality in VoIP," in Proceedings of the 2nd IEEE Internatioal Workshop on Haptic, Audio and Visual Environments and Their Applications (HAVE '03), pp. 49-54, Ottawa, Ontario, Canada, September 2003.

[8] J. Zhang, D. Yang, and Z. Quan, "Voice quality of VoIP in mobile communication systems," in Proceedings of the IEEE Radio and Wireless Symposium (RWS '06), pp. 131-134, San Diego, Calif, USA, January 2006.

[9] M. Narbutt and M. Davis, "Effect of free bandwidth on VoIP performance in 802.1lb WLAN networks," in Proceedings of the Irish Signals and Systems Conference (ISSC '06), pp. 123-128, Dublin, Ireland, June 2006.

[10] S. Choi, J. del Prado, N. S. Shankar, and S. Mangold, "IEEE 802.11 e contention-based channel access (EDCF) performance evaluation," in Proceedings of the IEEE International Conference on Communications (ICC '03), vol. 2, pp. 11511156, Anchorage, Alaska, USA, May 2003. 
[11] I. Dangerfield, D. Malone, and D. J. Leith, "Experimental evaluation of 802.11e EDCA for enhanced voice over WLAN performance," in Proceedings of the 4th International Symposium on Modeling and Optimization in Mobile, Ad Hoc and Wireless Networks, pp. 1-7, Boston, Mass, USA, April 2006.

[12] G. S. Paschos, I. Papapanagiotou, S. A. Kotsopoulos, and G. K. Karagiannidis, "A new MAC protocol with pseudo-TDMA behavior for supporting quality of service in 802.11 wireless LANs," EURASIP Journal on Wireless Communications and Networking, vol. 2006, Article ID 65836, 9 pages, 2006.

[13] Distributed Internet Traffic Generator, http://www.grid. unina.it/software/ITG/.

[14] P. Wang, H. Jiang, and W. Zhuang, "IEEE 802.11E enhancement for voice service," IEEE Wireless Communications, vol. 13, no. 1, pp. 30-35, 2006.

[15] Q. Ni, L. Romdhani, and T. Turletti, "A survey of QoS enhancements for IEEE 802.11 wireless LAN," Wireless Communications and Mobile Computing, vol. 4, no. 5, pp. 547-566, 2004.

[16] IEEE 802.11e-2005, "IEEE standard for information technology-telecommunications and information exchange between systems-local and metropolitan area networksspecific requirements-part 11: wireless medium access control (MAC) and physical layer (PHY) specifications: amendment 8: medium access control (MAC) quality of service (QoS) enhancements," November 2005.

[17] Z.-N. Kong, D. H. K. Tsang, B. Bensaou, and D. Gao, "Performance analysis of IEEE 802.11e contention-based channel access," IEEE Journal on Selected Areas in Communications, vol. 22, no. 10, pp. 2095-2106, 2004.

[18] ITU-T Rec. G.729, "Coding of speech at $8 \mathrm{kbit} / \mathrm{s}$ using conjugate-structure algebraic-code-exited linear prediction (CS-ACELP)," International Telecommunications Union, Geneva, Switzerland, 1996.

[19] ITU-T Rec. P.862.3, "Application guide for objective quality measurement based on recommendations P.862, P.862.1 and P.862.2," International Telecommunications Union, Geneva, Switzerland, 2005.

[20] ITU-T Rec. P.501, “Test signals for use in telephonometry," International Telecommunications Union, Geneva, Switzerland, 2000.

[21] P. Počta and M. Vaculík, "Method of choice of test signals for automatic intrusive measurement VTQoS," in Proceedings of the 4th International Conference on Measurement of Speech and Audio Quality in Networks (MESAQIN '05), Prague, Czech Republic, June 2005.

[22] P. Počta and M. Vaculík, "Determination of optimal test sequence for automatic intrusive measurement VTQoS on environment of fixed telecommunication network," in Proceedings of the 6th International Conference RTT, Ostrava, Czech Republic, 2005.

[23] P. Počta and M. Vaculík, "Researching of coders influence on basic measurement signals used in optimal test sequence," Advances in Electrical and Electronic Engineering, vol. 5, no. 3, pp. 377-380, 2006.

[24] P. Počta and M. Vaculík, "Comparison of test sequence for intrusive measurement of VTQoS with speech sequences in the environment of IP networks," in Proceedings of the 5th International Conference on Measurement of Speech and Audio Quality in Networks (MESAQIN '06), Prague, Czech Republic, June 2006.
[25] ITU-T Rec. P.830, "Subjective performance assessment of digital telephone-band and wideband digital codecs," International Telecommunications Union, Geneva, Switzerland, 1996.

[26] Wireshark network analyzer, http://www.wireshark.org/.

[27] A. W. Rix, J. G. Beerends, M. P. Hollier, and A. P. Hekstra, "Perceptual evaluation of speech quality (PESQ)-a new method for speech quality assessment of telephone network and codecs," in Proceedings of IEEE International Conference on Acoustics, Speech and Signal Processing (ICASSP '01), vol. 2, pp. 749-752, Salt Lake City, Utah, USA, May 2001.

[28] J. Holub, R. Šmíd, and M. Bachtík, "Child listeners as the test subject-comparison with adults and P.862," in Proceedings of the 2nd International Conference on Measurement of Speech and Audio Quality in Networks (MESAQIN '03), Prague, Czech Republic, May 2003. 

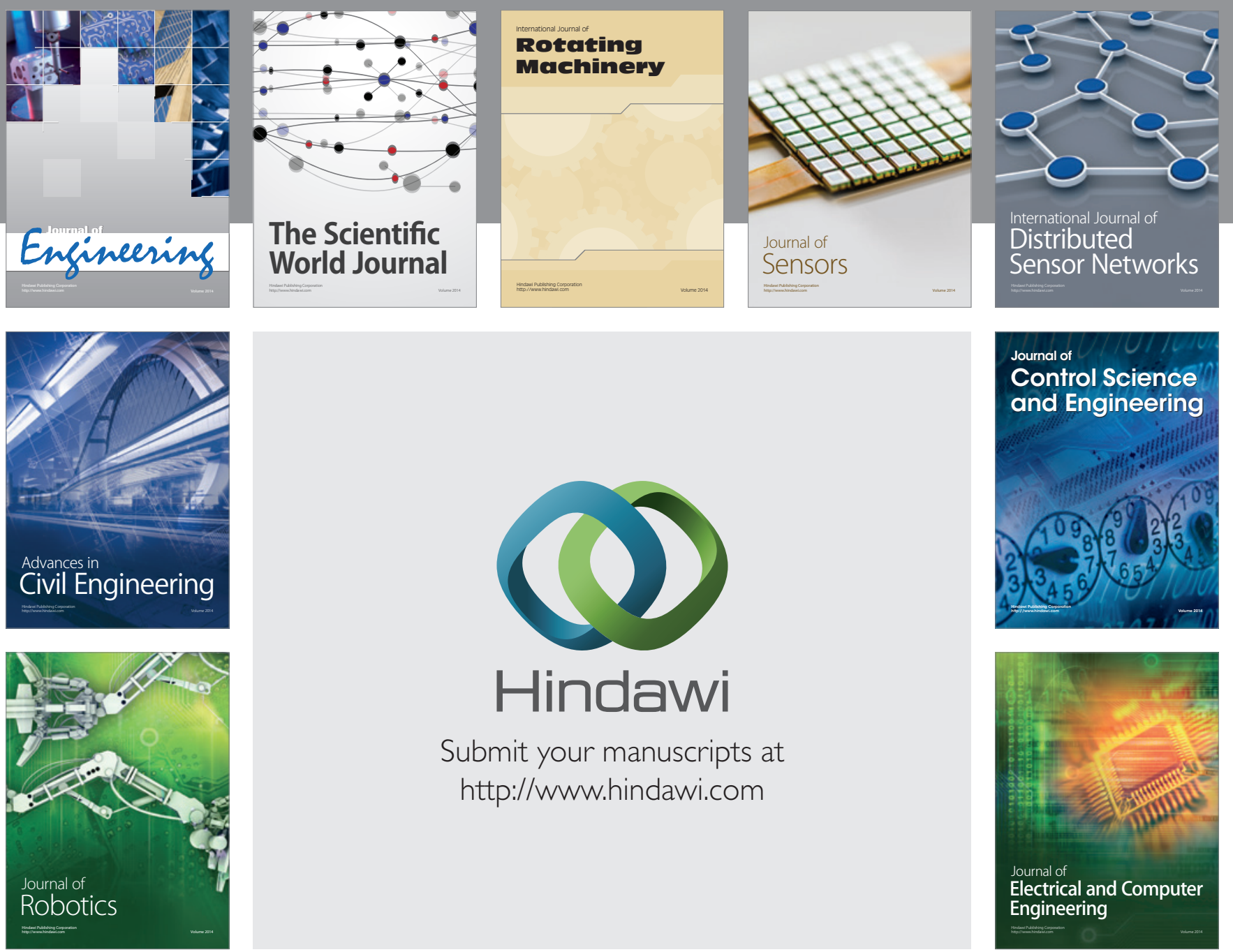

Submit your manuscripts at

http://www.hindawi.com
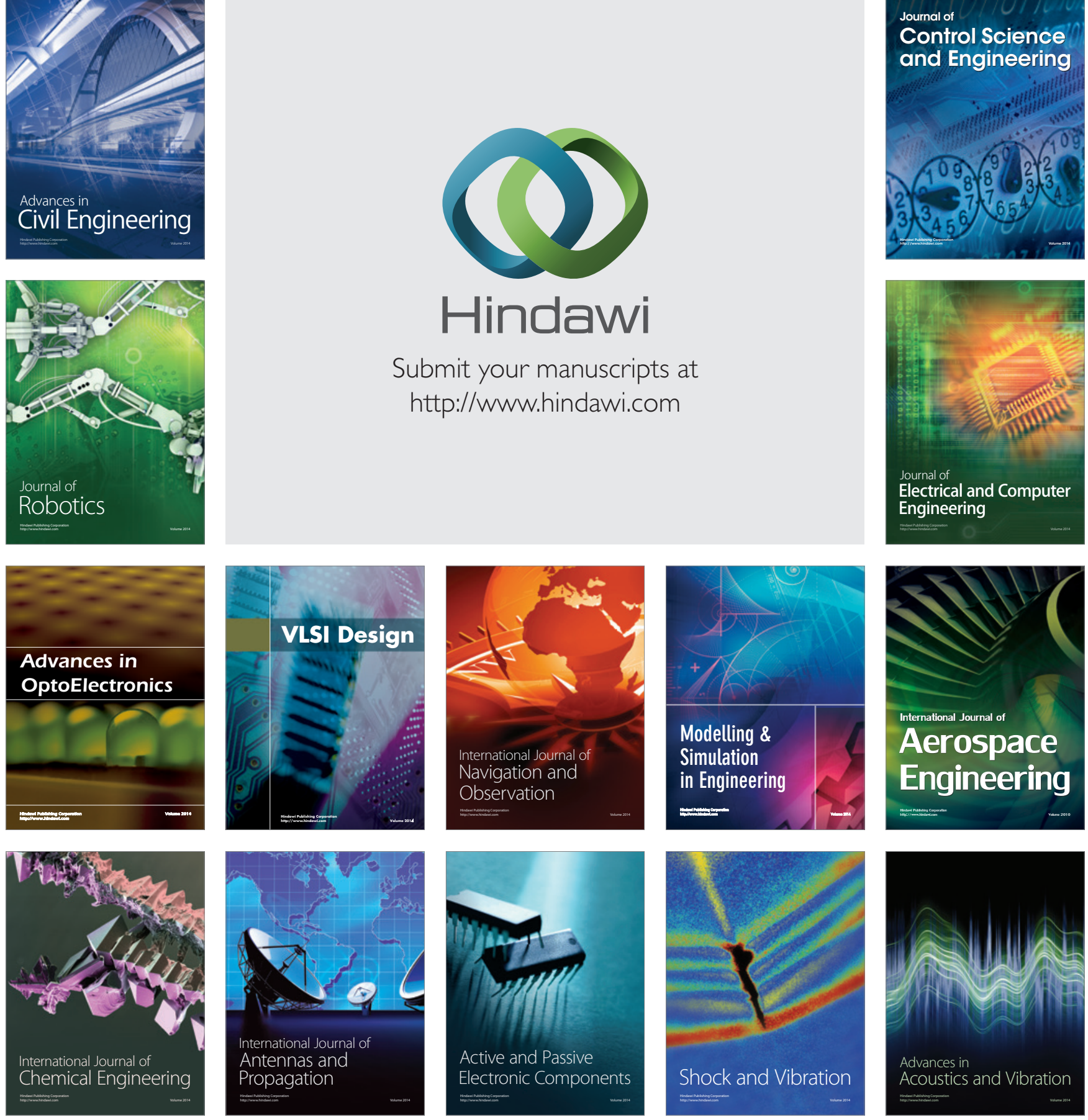